\title{
Standard of care in the surgical management of endometrial cancer
}

\author{
Kanishka Karunaratne ${ }^{1}$ \\ Sri Lanka Journal of Obstetrics and Gynaecology 2011; 33: 121-123
}

\section{Introduction}

Endometrial cancer is the second most common gynaecological cancer and the fifth most common female cancer overall, in the Western world. In the Asian continent, it ranks as the third common cancer in the female genital tract. Most women with endometrial carcinomas are post-menopausal, with the majority of cases occurring between fifty and sixty five years of age. Up to $25 \%$ of affected women are pre-menopausal and a minority (less than $5 \%$ ) is diagnosed before the age of 40 years. Endometrial cancer is associated with a number of reproductive risk factors (see Box) including early menarche and late menopause, continuous anovulation (PCOS), nulliparity and infertility.

\section{Box. Risk factors for endometrial cancer}

- Obesity

- Early menarche/late menopause

- Prolonged anovulation

- Polycystic ovarian syndrome

- Nulliparity

- Infertility

- Diabetes mellitus

- Hypertension

- Unopposed systemic oestrogen therapy

- Tamoxifen

- Oestrogen-secreting ovarian tumours

- Hereditary causes, e.g. HNPCC

\footnotetext{
${ }^{1}$ Consultant Gynae-oncologist, National Cancer Institute, Maharagama, Sri Lanka.

Correspondence: Kanishka Karunaratne

E-mail:kushank@sltnet.lk
}

Excessive unopposed endogenous oestrogen is common to all these clinical scenarios. Obese women are at increased risk of endometrial cancers. This is probably a result of increased conversion of adrenal androgens to oestrogens in peripheral fat. Levels of sex hormones-binding globulin are also reduced in obese women, further increasing the amount of bio available oestrogens. Occasionally, endometrial cancer may arise as a result of excessive endogenous oestrogen production by an ovarian granulosa cell tumour. Endometrial cancer may associate with diabetes and hypertension; although it is not clear whether these are simply markers of obesity or whether they are independent associations. It has long been accepted that the use of unopposed oestrogen replacement therapy increases the risk of endometrial cancer. Tamoxifen widely used in the treatment of breast cancer is an anti-oestrogenic drug that has weak oestrogenic effect on the female genital tract. Hereditary endometrial cancer accounts for less than 5\% of all endometrial cancers and is usually seen in association with hereditary non-polyposis colonic cancer syndrome (HNPCC). HNPCC is an autosomal dominant inheritant disorder. It is associated with a $40-60 \%$ life time risk of developing endometrial cancer and should be suspected in all women below the age of 45 presenting with endometrial cancer or where there is a long family history of bowel and gastric cancers.

\section{Clinical presentation}

Post menopausal bleeding is the commonest presenting symptom. Pre-menopausal women may present with post-coital or inter menstrual bleeding. In some cases change in menstrual pattern becomes increasingly heavy and erratic. Persistent vaginal discharge in a post-menopausal woman may indicate pyometra which means investigation to exclude cancer.

\section{Role of imaging in treatment planning}

A chest $x$ ray is required prior to surgery to detect metastasis, although these are rare in early-stage of endometrial disease. MRI is more sensitive than either ultrasound scan or computed tomography (CT) in the assessment of myometrial invasion. MRI may also be used for identifying extra uterine extension and cervical stromal involvement in which case more 
radical surgery can be considered. CT is used to evaluate upper abdomen especially in women with serous endometrial tumours in view of the tendency to transabdominal spread.

\section{Surgery for endometrial cancer}

Despite the use of preoperative imaging as an aid to treatment planning, staging of endometrial cancer remains surgicopathological. Full surgical staging used in FIGO system comprises total abdominal hysterectomy and bilateral salpingooophorectomy and peritoneal cytology and pelvic and paraaortic lymphadenectomy ${ }^{1}$. Many women do not, however undergo full surgical staging. Lymph node metastasis are present in less than $5 \%$ of cases of grade I and grade II endometrial tumours with less than 50\% myometrial invasion. Most gynaecologists, therefore accept that the risks and side effects of full surgical staging are not justified, given the low risk of nodal metastasis ${ }^{2}$. These women are suitable for surgery with extra-fascial TAH-BSO and peritoneal cytology. When clinical examination and /or preoperative imaging suggest cervical stromal involvement (stage II disease) radical hysterectomy, bilateral salpingo-oophorectomy and full pelvic lymphadenectomy are appropriate. If adequate surgical margins are achieved and the nodes are free of disease, the procedure is considered curative and adjutant radiotherapy is not indicated ${ }^{3}$.

\section{Role of extended surgical staging}

The overall 5-year survival rate following treatment for endometrial carcinomas is appropriately $75-80 \%{ }^{4}$. This reflects early presentation in the majority of cases, although five year survival falls rapidly as stage increases (Table 1). Approximately $8-10 \%$ of women with the disease, apparently confined to the uterus have occult nodal disease. It has therefore being surgical practice to selectively perform lymphadenectomy in addition to TAH-BSO for those women with high risk of lymph node metastasis based on pre operative assessment of depth of myometrial invasion and / or grade ${ }^{5}$. There has been considerable debate regarding the role of lymphadenectomy in endometrial cancer surgery. ASTEC trial (a study in the treatment of endometrial cancer) has shown no significant difference in the overall survival between those women who had lymphadenectomy and those who did not. These results clarify the role of lyphadenectomy and confirm that there is no therapeutic benefit for routine lymphadenectomy in women with low risk endometrial cancers. The risk of occult extra uterine metastasis is higher for non endometrioid tumours and extended surgical staging should be undertaken. An omental biopsy is taken in cases of serous endometrial carcinoma in view of the risk of omental metastasis.
Table 1. Approximate five-year survival according to the FIGO stage

\begin{tabular}{cc}
\hline Stage & Approximate five-year survival (\%) \\
\hline I & 85 \\
II & 75 \\
III & 45 \\
IV & 25 \\
\hline
\end{tabular}

\section{Minimal access surgery}

Laparoscopic surgery is increasingly used in the management of endometrial cancers. Both laparoscopic total hysterectomy and laparoscopically assisted vaginal hysterectomy are feasible procedures and when successfully performed complication rates are minimum specially in elderly and obese women. Both pelvic and para aortic lymphadenectomy with omental sampling can be safely and adequately performed in the management of high-grade disease. Laparoscopic surgery for endometrial cancer should be undertaken by appropriately trained surgeons. In retrospective studies, endometrial cancer, recurrence rates have been found to be similar in both procedures: laparoscopy and open surgery. Wound break down, blood loss and infections appear to be lower when laparoscopic techniques are used.

\section{Vaginal hysterectomy}

Occasionally an abdominal hysterectomy is not possible due to severe co-morbidities. In patients with morbid obesity abdominal hysterectomy may be associated with more complications. In these situations vaginal hysterectomy is the treatment of choice.

\section{Complications of surgery}

Complications related to surgery for endometrial cancer may be specifically related to the surgical procedure itself or may be due to complications of anaesthesia, thromboembolism and infections. Severe late complications of surgery for endometrial cancer are uncommon.

\section{References}

1. Boronow RC, Morrow CP, Creasman WT, et al. Surgical staging in endometrial cancer: clinical-pathological finding of a prospective study. Obstetric and Gynaecology 1984; 63: 835-42. 
2. Calais G, Descamps L, Vitu L, et al. Lymphadenectomy in the management of endometrial cancer stage I and II. Retrospective study of 155 cases. Clinical Oncology 1990; 2: 318-23.

3. Poulsen HK, Jacobsen M, Bertelsen K, et al. From the Danish Endometrial Cancer Group (DEMCA). Adjuvant radiation therapy is not necessary in the management of endometrial carcinoma stage I, low-risk cases. International Journal of Gynaecological Cancer 1996; 6: 38-43.
4. COSA-NZ-UK Endometrial Cancer Study Group. Pelvic lymphadenectomy in high risk endometrial cancer. International Journal of Gynaecological Cancer 1996; 6: 102-7.

5. Grigsby PW, Perez, CA, Kuten A. Prognostic factor for local control and distant metastasis and implications of the new FIGO surgical staging system. International Journal of Radiation, Oncology, Biology, Physics 1992; 22: 905-11. 